\title{
Root-Mean-Square Dipole Moment and Neutron Scattering Function of 18-Crown-6 in Cyclohexane: Comparison of Three Potential Models
}

\author{
F. T. H. Leuwerink* and W. J. Briels \\ Chemical Physics Laboratory, University of Twente, P.O. Box 217, 7500 AE Enschede, The Netherlands
}

Received: May 1, 1995; In Final Form: August 3, $1995^{\circledR}$

\begin{abstract}
Molecular dynamics simulations have been performed in order to study the effect of a new charge density distribution for 18-crown-6, recently described in the literature [J. Mol. Struct. (THEOCHEM) 1994, 305, 249], on different properties of the crown ether. Comparisons are made with results discussed in a previous paper where other potential models were employed. Remarkable shifts in the spectrum of conformations are observed, and for the first time an average dipole moment is calculated that is in good agreement with experiment. We have calculated neutron scattering cross sections and X-ray intensities for two potential models. Of these, only the neutron scattering cross section turned out to be sensitive to the differences in the structures resulting from these potentials.
\end{abstract}

\section{Introduction}

Over the years macrocyclic polyethers (crown ethers) ${ }^{1.2}$ have received widespread attention in many fields of chemistry. Much of this interest results from their remarkable ability to bind cations selectively and transport them eventually into a lipophilic environment. Nowadays there is a wealth of crystallographic ${ }^{3}$ and thermodynamic ${ }^{4.5}$ data available for numerous complexes. Crown ethers are also of great interest to theoretical chemists because they represent the simplest model system which might contain some of the features of enzyme specificity in their interactions with ions and neutral molecules.

The 18-crown- 6 molecule is the best known representative of the great family of crown ethers. It is one of the simplest crown ethers and has been the focus of many statistical mechanical ${ }^{6-12}$ and $a b$ initio $^{13-17}$ studies. Previous theoretical investigations ${ }^{18-22}$ have shown that 18 -crown- 6 is a very flexible molecule that can adopt many different conformations within a narrow energy range. The role of crown ethers as catalyst can be related to this flexibility. An important property connected with their complexation and catalytic power is the dipole moment. The various conformations adopted by 18 -crown- 6 exhibit very different dipole moments. Up until now the average dipole moments that were calculated from either Monte Carlo or molecular dynamics simulations were in poor agreement with experiment.

In a previous paper, ${ }^{23}$ we investigated structural features and the dipole moment of 18 -crown- 6 in a cyclohexane solution and in vacuo by means of molecular dynamics simulations. We came to the conclusion that conformational statistics are very much alike in the nonpolar solvent and in the gas phase. The dipole moments we calculated from the simulations were comparable with previously published theoretical results, ${ }^{11.22}$ but differed appreciably from the experimental value. ${ }^{24.25}$ After a careful theoretical investigation, we proposed a new expression relating the mean square dipole moment to the experimental data and found an even larger discrepancy between theory and experiment than was obtained from a conventional analysis.

In this paper we investigate a new set of charges for 18crown- 6 that was recently published. ${ }^{32}$ We focus on the average

\footnotetext{
${ }^{\otimes}$ Abstract published in Advance ACS Abstracts, September 15, 1995.
}

dipole moment and the distribution of its conformations. We compare the results of long MD simulations of the molecule in the gas phase and in cyclohexane with those of the simulations we discussed in ref 23 . For two of the potential models neutron and $\mathrm{X}$-ray scattering functions are determined. In the following section we first give some background on the different sets of charges that were used by us and other groups. In section 3 the methods and procedures are described. In section 4 the results are discussed, and the conclusions are finally given in section 5 .

\section{Background}

In simple molecular force fields, the molecular electron density is usually modeled by point charges fixed on welldefined sites in the molecular frame. Polarization effects occurring when a molecule adopts different geometries are not treated explicitly, but only by using effective charges.

For the 18-crown-6 several sets of charges exist. Wipff $e t$ al. ${ }^{18}$ determined two sets of charges for the crown ether molecule. To study cation specificity, they determined charges from calculations on complexes of sodium and potassium with dimethyl ether. They varied the charge on the oxygen atom in dimethyl ether and the nonbonded parameters of $\mathrm{Na}^{+}$and $\mathrm{K}^{+}$ to fit the experimental $\Delta H$ of complexation and metal-oxygen distances of the $\mathrm{M}^{+}$-water complexes, assuming that these latter properties are the same in $\mathrm{M}^{+}-$dimethyl ether complexes and in $\mathrm{M}^{+}$-water complexes. The reproduction of the experimental data required $q_{0}$ to be equal to $-0.6 e$. For the crown itself Wipff $e$ t al. stated that a charge on oxygen of $-0.3 e$ seemed most appropriate, attributing the remaining $-0.3 e$ to polarization by the $\mathrm{M}^{+}$ions. There was no charge on the hydrogen atom. This set of charges was for instance used in refs $9,11,26$, and 27.

Slightly different charges were used in several MD studies, ${ }^{20.28 .29}$ employing the united atom approach. The charges were selected so as to reproduce the dipole moment of dimethyl ether. This required $q_{0}$ to be equal to $0.34 e$. Mazor et al. ${ }^{28}$ investigated a set of charges with $q_{0}$ equal to $-0.6 e$ for the complexed crown ether in vacuum. They found that the calculated thermodynamic properties were qualitatively similar to the ones obtained with $q_{0}=-0.34 e$ but quantitatively in poorer agreement with experiment. 
TABLE 1: Potential Models Used in the Different MD Simulations

\begin{tabular}{lrrr}
\hline & \multicolumn{3}{c}{ charge (in $e$ ) on } \\
\cline { 2 - 4 } \multicolumn{1}{c}{ simulation } & $\mathrm{O}$ & \multicolumn{1}{c}{$\mathrm{C}$} & \multicolumn{1}{c}{$\mathrm{H}$} \\
\hline in vacuo I and solution (ref 23) & -0.3000 & 0.1500 & 0.0000 \\
in vacuo II (ref 23) & -0.4060 & 0.2440 & -0.0205 \\
in vacuo III and solution (this study) & -0.4418 & -0.0079 & 0.1144
\end{tabular}

A second class of charge sets were taken from $a b$ initio calculations. Kowall and Geiger, ${ }^{12}$ also employing the united atom approximation, used $q_{0}=-0.4 e$ suggested by $a b$ initio calculations in ref 30 . In ref 30 , the polarization effect due to complexation with $\mathrm{K}^{+}$was evaluated as $0.05 e$, in accordance with the results of ref 14 . This means that the polarization effect has been largely overestimated by Wipff $e t$ al.

Other molecular dynamics studies ${ }^{6.8 .10 .21 .22}$ used point charges at the 18-crown- 6 atoms obtained by fitting point charge models to $a b$ initio electrostatic potentials of small fragments of the molecule; the $a b$ initio calculations were done at the HartreeFock/6-31G* level. ${ }^{31}$ This resulted in a charge on oxygen of $-0.406 e$ and a small negative charge of $-0.021 e$ on hydrogen.

Recently, charges were determined from $a b$ initio calculations at different levels of approximation, using seven different conformations of the molecule. ${ }^{32}$ The relative energies of these seven conformers were used as data in a fitting procedure to determine the charges in several empirical force fields. The most sophisticated calculations included electron correlation via full second-order Møller-Plesset perturbation theory and employed the $6-31 \mathrm{G}$ basis set. The resulting charges show a remarkable shift of electron density from hydrogen to carbon compared to the charges obtained from calculations on fragments of the macrocycle..$^{31}$ With these latter charges, the authors found that empirical force field calculations reproduced the $a b$ initio relative energies at the Hartree-Fock/STO-6G level, which is known to underestimate the repulsive electrostatic energy.

In this study we use the charges from ref 32 determined at the MP2(full) level, for a molecular dynamics simulation of 18crown- 6 in vacuum and in cyclohexane. We compare the results of the gas phase simulation, which will be referred to as simulation III, with the results of in vacuo simulations I and II of ref 23. We also compare the results of the solution described here with the one of ref 23 . The force field parameters only differ in the sets of charges used (see Table 1).

\section{Methods and Procedures}

Two simulations were performed with the GROMOS package: ${ }^{33}$ a simulation of an isolated 18-crown- 6 molecule in the NVE ensemble and a simulation of a cyclohexane solution at constant $N p T$.

The computational details are the same as in ref 23 , except that arbitrary conformations were chosen as starting structures for the 18-crown-6 molecule instead of the one with $D_{3 d}$ symmetry. For the cyclohexane solution, six different starting configurations were produced. After the equilibration, each box was run for $250 \mathrm{ps}$. The total simulation time for the gas phase run amounted to $30 \mathrm{~ns}$; that for the solution amounted to 1.5 ns. The non-Coulombic parameters for the crown ether were again taken from the AMBER all-atom force field. ${ }^{34}$

We used the charges from ref 32 that were optimized for scaling factors equal to 1.0 for the electrostatic and 0.5 for the van der Waals $1-4$ interactions, which are the same scaling factors we applied in all our simulations.

Each methylene unit in a cyclohexane molecule was treated as a single force center with appropriate mass. The parameters for this solvent were taken from Harris and Stillinger, ${ }^{35}$ who
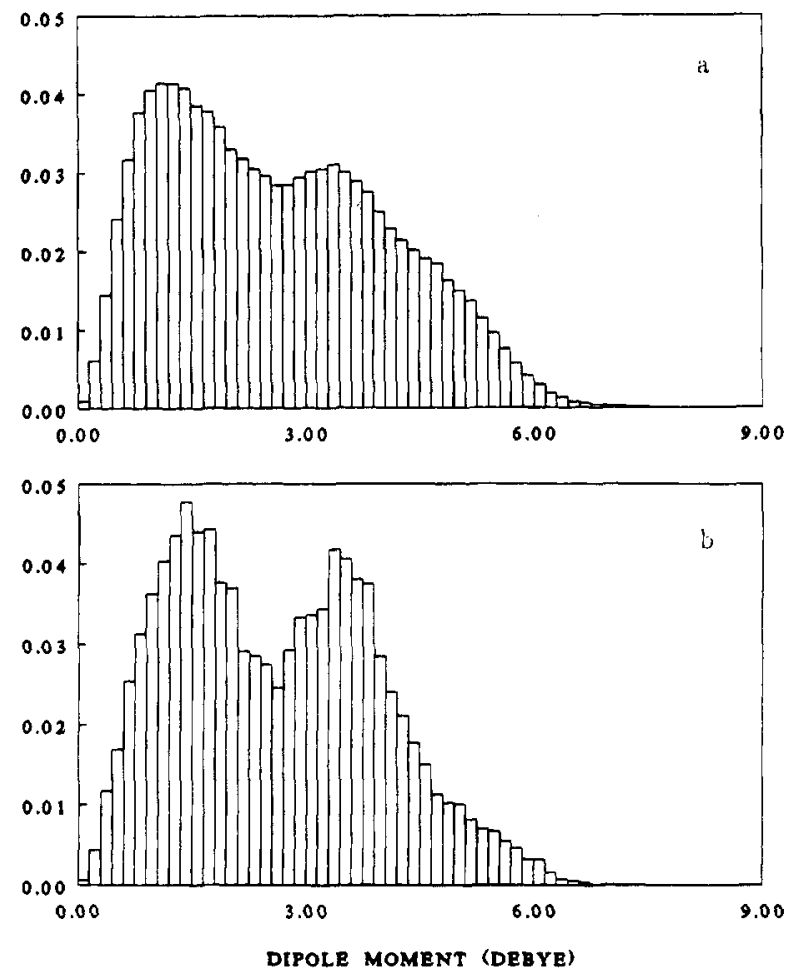

Figure 1. Normalized distributions of the dipole moment of 18-crown6: (a) in vacuo simulation; (b) cyclohexane solution.

studied the liquid structure of cyclohexane. The parameters of their revised Lennard-Jones and torsional contribution were adapted to the form of the potential energy function of GROMOS

The computational boxes for the cyclohexane solution were prepared with all solvent molecules in the chair conformation. Calculation has shown that the twisted boat isomer lies 28.6 $\mathrm{kJ} / \mathrm{mol}$ (experiment: $23 \mathrm{~kJ} / \mathrm{mol}$ ) in energy above the chair minimum. ${ }^{35}$

\section{Results and Discussion}

4.1. Conformational Sampling and the Dipole Moment. During the gas phase simulation 2927 symmetry-unique conformations were sampled, notably fewer than the 5628 and 3568 sampled during respectively simulations I and II of ref 23 . (Notice that the total simulation time for simulation II was only half of that for the other two.) At the same time the distribution of conformations has become much narrower. The 29 conformers (Table VIIS in the supporting information) which had an occurrence of $0.5 \%$ or more contributed to $66.49 \%$ of the total, to be compared with $34.73 \%$ and $40.68 \%$ for simulations I and II. (The conformations from simulations I and II with an occurrence of $0.5 \%$ or more together with their average dipole moment are given in respectively Tables VS and VIS in the supporting information.)

During the simulation of the solution, 228 symmetryindependent conformations were sampled. Those with a frequency of $0.5 \%$ or more (31) constituted $89.35 \%$ of the total. Compared with the $2 \mathrm{~ns}$ MD run of the solution of ref 23 , we again see that the distribution of conformers has become more compact.

Figure 1 shows the statistics of the dipole moments for both the vacuum and the solution. Two peaks are observed at respectively 1.2 and $3.4 \mathrm{D}$. For simulation II of ref 23 we also observed two peaks (at 0.8 and $2.5 \mathrm{D}$ ). With potential model I the structures were distributed over a much broader range. 
TABLE 2: Conformations of Ref 38 and Their Occurrences in the Different Gas Phase Simulations

\begin{tabular}{|c|c|c|c|c|c|}
\hline \multirow[b]{2}{*}{ conformation } & \multirow[b]{2}{*}{$(\mathrm{sym})$} & \multirow[b]{2}{*}{ rel energy ${ }^{a}$} & \multicolumn{3}{|c|}{ \% occurrence (no. on freq list) from in vacuo simulation } \\
\hline & & & III $^{b}$ & $\mathrm{II}^{b}$ & $I^{b}$ \\
\hline $\mathbf{I}$ & $\left(C_{i}\right)$ & 0.0 & $12.861(1)$ & $0.143(138)$ & $0.010(1004)$ \\
\hline II & $\left(C_{2}\right)$ & 10.3 & $0.739(22)$ & & \\
\hline III & $\left(C_{1}\right)$ & 11.0 & $0.541(26)$ & $0.009(983)$ & $0.001(3296)$ \\
\hline IV & $\left(C_{2}\right)$ & 11.7 & $1.126(19)$ & $0.014(737)$ & $0.001(3123)$ \\
\hline V & $\left(C_{1}\right)$ & 11.9 & $1.666(14)$ & & \\
\hline VI & $\left(C_{i}\right)$ & 13.9 & $0.017(354)$ & $10.667(1)$ & $5.903(1)$ \\
\hline VII & $\left(C_{1}\right)$ & 15.4 & $0.190(61)$ & $0.141(140)$ & $0.016(779)$ \\
\hline VIII & $\left(C_{1}\right)$ & 15.6 & $0.488(30)$ & $0.038(368)$ & \\
\hline IX & $\left(S_{6}\right)$ & 17.2 & $0.168(74)$ & & $0.003(1989)$ \\
\hline $\mathbf{X}$ & $\left(C_{1}\right)$ & 18.2 & $0.006(652)$ & & $0.011(957)$ \\
\hline XI & $\left(C_{1}\right)$ & 18.7 & $0.295(47)$ & & \\
\hline XII & $\left(C_{1}\right)$ & 19.4 & $0.018(336)$ & & \\
\hline
\end{tabular}

${ }^{a}$ In kJ mol ${ }^{-1}$. Taken from ref $38 .{ }^{b}$ The total number of symmetry-unique conformations is 2927,3568 , and 5628 for respectively in vacuo simulations III, II, and I.

The dipole moments were found to be distributed more or less Gaussian (see Figure 1 of ref 23).

With the charges of Szentpály and Shamovsky, we calculated for the 18-crown-6 molecule an average dipole moment of 3.02 $\mathrm{D}(\mathrm{SD}=0.45 \mathrm{D})$ from the vacuum simulation and a value of $2.94 \mathrm{D}(\mathrm{SD}=0.87 \mathrm{D})$ from the cyclohexane solution. These values are considerable larger than the 1.98 and $2.39 \mathrm{D}$ from respectively in vacuo simulations I and II or the $2.04 \mathrm{D}$ for the crown ether in cyclohexane reported in our previous paper. In our previous paper we stated that electronic polarizability might be important and could perhaps be responsible for the discrepancy between theory and experiment. The set of charges under investigation in this paper have been obtained by fitting the energies of several different conformations of the crown ether and may be expected to partially include the effects of intramolecular polarization. Indeed, the dipole moments obtained with this potential are in much better agreement with experimental results and are in between the value of $2.76 \mathrm{D}$ reported by Caswell and Suvannunt ${ }^{25}$ and $3.17 \mathrm{D}$ we obtained from the experimental data with an expression we derived in ref 23 (eq 16). Including the polarizability of the solvent in the simulation might reduce the difference between the theoretical value and the value calculated from our expression even more.

Although the above results are rather satisfactory, two critical remarks should be made at this point. First, if the present charges are used to calculate the dipole moment of dimethyl ether, a value of $2.14 \mathrm{D}$ results. (The charges on the hydrogen atoms were taken to be $2 / 3 \times 0.1144$ e.) This result is a much larger than the value of $1.30 \mathrm{D}$ reported for dimethyl ether in the gas phase. ${ }^{36}$ Unfortunately, we have not been able to find experimental values of the dielectric constant of liquid dimethyl ether, from which an effective dipole moment in the liquid state might have been calculated. Doing so for diethyl ether an effective dipole moment of $1.40 \mathrm{D}$ for the liquid state is found compared to $1.15 \mathrm{D}$ for the gaseous state. ${ }^{37}$ For dimethyl ether polarization effects in the liquid state are expected to be of approximately the same magnitude. Second, we notice that Szentpály and Shamovsky apparently have not performed geometry optimization at the same level as their various quantum mechanical calculations. This may have resulted in an overestimation of polarization effects.

In ref 23 we concluded that conformational statistics was almost the same in vacuo and in the apolar cyclohexane. In the following subsection, where the conformations will be examined in more detail, we will therefore mainly concentrate on the three vacuum simulations.

4.2. Comparison of the Conformational Spectra of the in Vacuo Simulations. Szentpály et al. ${ }^{38}$ predicted a number of low-energy conformations generated with a "slow-cooling" Monte Carlo technique. The charges they used were optimized for a van der Waals $1-4$ scaling factor of 1.0 and differed a little from those that were used in this study. In Table 2 these conformations are given, together with their statistical importance from the different gas phase simulations. The first thing to be noticed from Table 2 is that the lowest energy conformation, which has $C_{i}$ symmetry, is also the most frequently sampled conformer from in vacuo simulation III. The crystallographic $C_{i}$ conformation (VI) on the other hand, which is the conformation adopted by uncomplexed 18-crown- 6 in the solid state and which was the most occurring structure during simulations I and II $(5.903 \%$ and $10.667 \%$, respectively; see Tables VS and VIS), appeared during simulation III only with a very low frequency $(0.017 \%)$. With potential models I and II this conformation corresponds to the global energy minimum. Similarly, the crystallographic $D_{3 d}$ conformation, which with previous force fields was predicted to be very stable and which was sampled during simulation I (structure 8 in Table VS), but not during simulation $\Pi$, was also not observed during simulation III. The $D_{3 d}$ arrangement is found in crystals with metal ions as well as in complexes of the crown with polar molecules. Szentpály et al. stated that the crystallographic $C_{i}$ conformation may very well not be the global minimum in vacuo and that the $D_{3 d}$ symmetry is very unstable for uncomplexed 18-crown-6 in the gas phase. With the present force field, we calculated relative energies of 15.2 and $86.0 \mathrm{~kJ} / \mathrm{mol}$ with respect to the $C_{i}$ conformation for respectively the crystallographic $C_{i}$ and $D_{3 d}$ structure. Although it is not impossible that packing effects make the crown crystallize in a structure different from the global gas phase minimum, $15.2 \mathrm{~kJ} / \mathrm{mol}$ seems to be rather large to be compensated for, especially in view of the fact that there are no hydrogen bonds or other specific intermolecular interactions available to do so.

The most populated conformations from simulation III have low symmetry (Table VIIS). Structure 1 has $C_{i}$ symmetry; the others are either $C_{2}$ or $C_{1}$ point-group molecules with the exception of form 3 , which is a $C_{3}$ structure. Structure VII (Table 2) generated by Szentpály et al. shows close resemblance with the $C_{3}$ conformer. They differ in only two dihedral angles. We calculated an energy of only $2.2 \mathrm{~kJ} / \mathrm{mol}$ for this $C_{3}$ conformation relative to the $C_{i}$ conformation. It was the most occurring structure in the simulation of the solution $(17.446 \%)$. The $C_{i}$ structure was third most frequently sampled $(6.733 \%)$, and form $2(14.460 \%)$ was the same as in the vacuum simulation.

Szentpály et al. noted that the conformations they had generated showed a tendency to fill the inner space of the crown ether by hydrogen atoms. In order to check this, we calculated 
TABLE 3: Average Hydrogen-Center of Mass Distances ${ }^{a}$ for the 18-Crown-6 Molecule from the Different MD Simulations ${ }^{b}$

\begin{tabular}{lccc}
\hline & \multicolumn{3}{c}{ potential model } \\
\cline { 2 - 4 } & I & II & III \\
\hline in vacuo & $3.82(0.91)$ & $3.75(0.96)$ & $3.60(1.04)$ \\
solution & $3.80(0.91)$ & & $3.59(1.03)$
\end{tabular}

a In $\AA .{ }^{b}$ Standard deviations are given in parentheses.

the average distance of the hydrogen atoms to the center of mass of the molecule (Table 3 ). In Figure 5 the distributions of these distances are given for all the performed simulations. The difference between the results for potential models I and II are not very large, and those for model III deviate more compared to the former two. There is indeed in general a stronger tendency with the charge distribution of model III to fill the cavity of the crown ether molecule. For the crystallographic $C_{i}$ conformation and the $C_{i}$ symmetry from simulation III, we respectively found an average distance of 3.83 and $3.59 \AA$. More and stronger intramolecular hydrogen bridges explain the change in the stability of these structures with respect to each other in going from potential I to III.

Next, the populations of the conformations are characterized by means of the inertia tensor as described in ref 23 . In Figures $2-4$ the densities are given for the projection of the positions of the oxygen and carbon atoms on the $x y, x z$, and $y z$ planes for respectively in vacuo simulations I, II, and III. The above planes are defined by the eigenvectors of the inertia tensor.

The density plots from gas phase simulations I and II show some remarkable similarities. The density plots from simulation III deviate more. The potential models used in the first two simulations mainly differ by an enhanced charge on oxygen and carbon for model II (see Table 1). The charge on hydrogen is hardly affected. Potential model III distinguishes itself from the other two by a large shift of charge density from hydrogen to carbon, which apparently results in a pronounced change in the spectrum of conformations. These findings are in accordance with the average principal moments of inertia given in Table 4, together with the principal moments for the $C_{i}$ and $C_{3}$ conformation (respectively forms 1 and 3 in Table VIIS). The average values for simulations I and II lie closer to each other than they are to those of simulation III. The discrepancies, however, are small. We mark that the $C_{i}$ conformation is rather similar to the crystallographic $C_{i}$ structure, which is only a local minimum in the spectrum of conformations of simulation III, in the sence that it has a nearly identical distribution of its atom masses. Remarkably, both conformations can be converted into each other by shifting the torsional angles by one bond.

From Table 4 we see that in all performed simulations the average shape of the 18-crown-6 molecule is elliptical. The larger differences between the average principal moments of the gas phase and the solution employing potential model III in comparison with the corresponding simulations using potential model I are due to the large contribution of the $C_{3}$ conformation in the former solution. The molecule in vacuo and in cyclohexane (apolar solvents) does not possess a clear cavity like the $D_{3 d}$ conformation, being the general structure of the complexed macrocycle. The stronger tendency of potential III to fill the cavity with hydrogens results, among other conformers, in the very stable $C_{3}$ conformation which differs from the $D_{3 d}$ symmetry in that three hydrogen atoms are folded toward the center of the ring. The strong interactions in complexes between guest and host largely offset the energy needed to organize the crown ether, making the oxygens accessible in the process of complexation. ${ }^{8}$

In ref 23 we found that strong correlations existed between the localized peaks in the density plots, and we were able to classify the many conformations into a small number of groups. It was clear that these groups contained many different structures which showed remarkable structural similarities. This is not done here, but looking at the most populated conformations (Table VIIS), we see that most forms resemble to a certain extent the $C_{i}$ or the $C_{3}$ structure.

4.3. Molecular Volume of 18-Crown-6 in Cyclohexane. In ref 23 employing potential model I for the crown ether, a partial molar volume of $288.3 \AA^{3}$ for the crown ether in cyclohexane was calculated. This value differed appreciably from the volumes reported by Letcher et al. ${ }^{39.40}$ who measured volumes in the range from $364.0 \AA^{3}$ in the very polar acetonitrile to $390.7 \AA^{3}$ in the nonpolar $\mathrm{CCl}_{4}$. At the time we determined the density of liquid 18-crown-6 to check whether our value could be the result of a deficient force field. The value from the simulation compared rather well with the experimental density. (A $4.5 \%$ difference was found.) Also, the calculated density for pure cyclohexane was within a few percent of the experimental value.

From the simulation performed in this study, a value of 321.6 $\AA^{3}$ emerged. Assuming that the value of the molecular volume in cyclohexane will not be very different from that in apolar carbon tetrachloride, a value of $321.6 \AA^{3}$ is still not very good. We therefore determined properties that when compared with experimental results could give more information about the better potential for the crown ether molecule. This is discussed in the following subsection.

4.4. Radial Distribution Functions and Scattering Spectra. First, we look shortly at the dipole moment again. The average dipole moments in section 4.1 were calculated as the square root of the mean square of the dipole moment: $\left\langle u^{2}\right\rangle^{1 / 2}$. Using $\left\langle\vec{r}_{i} \cdot \vec{r}_{j}\right\rangle=r_{i}{ }^{2}+r_{j}{ }^{2}-1 / 2\left\langle r_{i j}{ }^{2}\right\rangle,\left\langle\mu^{2}\right\rangle$ can be written as

$$
\begin{aligned}
\langle\vec{\mu} \cdot \vec{\mu}\rangle & =-1 / 2 \sum_{i} \sum_{j} q_{i} q_{j}\left\langle r_{i j}{ }^{2}\right\rangle \\
& =-1 / 2 \sum_{i} \sum_{j} q_{i} q_{j} S_{i j} \int_{0}^{\infty} \mathrm{d}^{3} r g_{i j}(r) r_{i j}{ }^{2}
\end{aligned}
$$

where $S_{i j}$ represents the number of atom pairs $i, j, q_{i}$ is the charge

\begin{tabular}{|c|c|c|c|c|c|}
\hline \multirow[b]{2}{*}{ in vacuo simulations } & \multirow[b]{2}{*}{$C_{i}$} & \multirow[b]{2}{*}{$C_{3}$} & \multicolumn{3}{|c|}{ potential model } \\
\hline & & & I & II & III \\
\hline$x$ axis & 17.510 & 25.887 & $20.032(2.407)$ & $19.899(2.360)$ & $20.723(2.471)$ \\
\hline$y$ axis & 34.970 & 25.942 & $30.647(2.613)$ & $31.078(2.963)$ & $30.595(2.874)$ \\
\hline $\begin{array}{l}z \text { axis } \\
\text { solutions }\end{array}$ & 51.026 & 50.329 & $47.519(2.983)$ & $47.658(3.444)$ & $48.684(2.393)$ \\
\hline$x$ axis & & & $19.781(2.416)$ & & $21.484(2.664)$ \\
\hline$y$ axis & & & $30.277(2.584)$ & & $29.311(2.643)$ \\
\hline$z$ axis & & & $47.100(2.202)$ & & $48.440(2.099)$ \\
\hline
\end{tabular}

TABLE 4: Average Principal Moments of Inertia $\left(\times 10^{45} \mathrm{~kg} \mathrm{~m}^{2}\right)^{a}$

"Standard deviations are given in parentheses. 

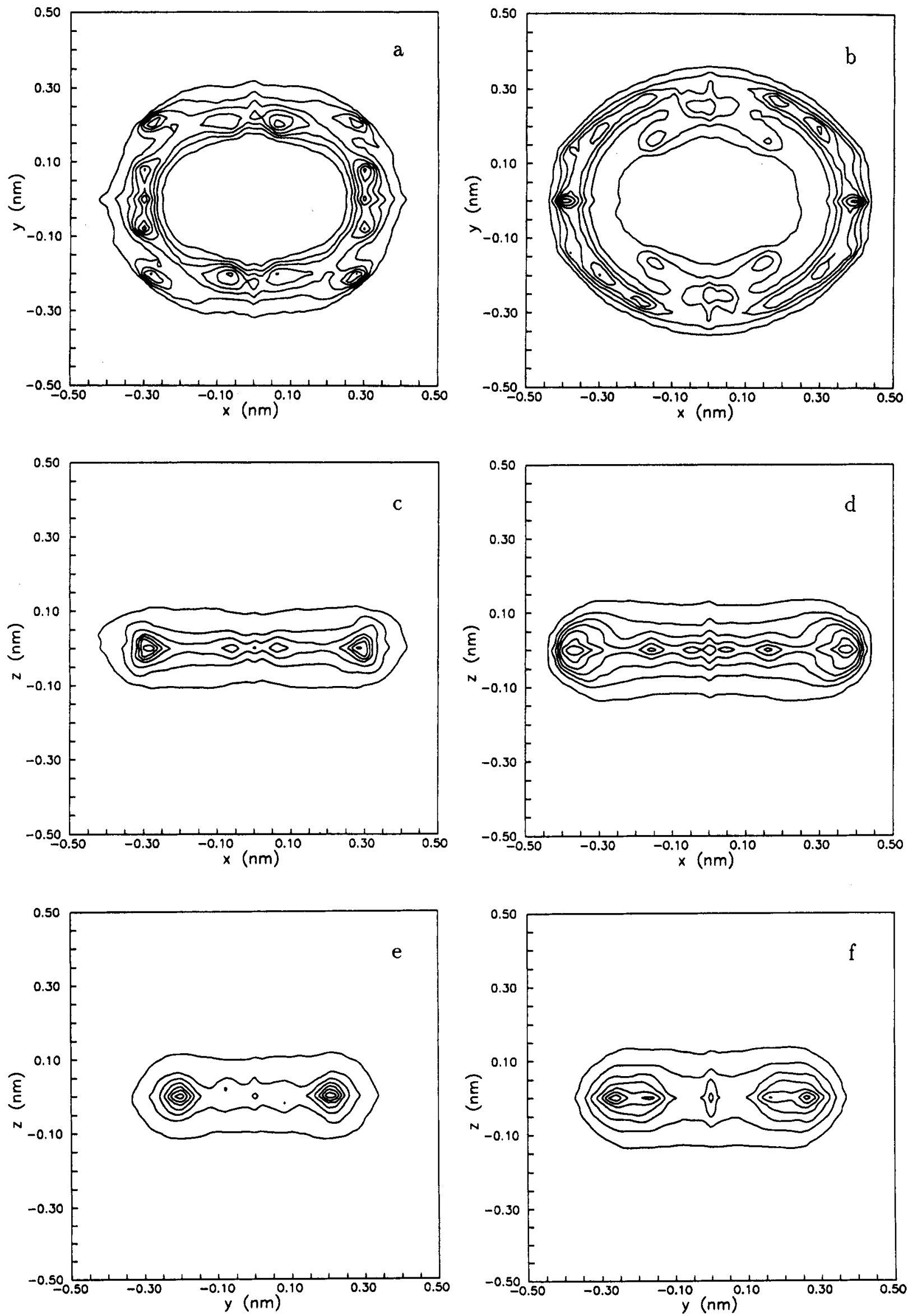

Figure 2. Contour plots for in vacuo simulation I: (a) projection of the oxygen atoms of the crown ether molecule on the $x y$ plane; (b) projection of the carbon atoms on the $x y$ plane; (c) projection of the oxygen atoms on the $x z$ plane; (d) projection of the carbon atoms on the $x z$ plane; (e) projection of the oxygen atoms on the $y z$ plane; (f) projection of the carbon atoms on the $y z$ plane (data from ref 23). 
16554 J. Phys. Chem., Vol. 99, No. 45, 1995
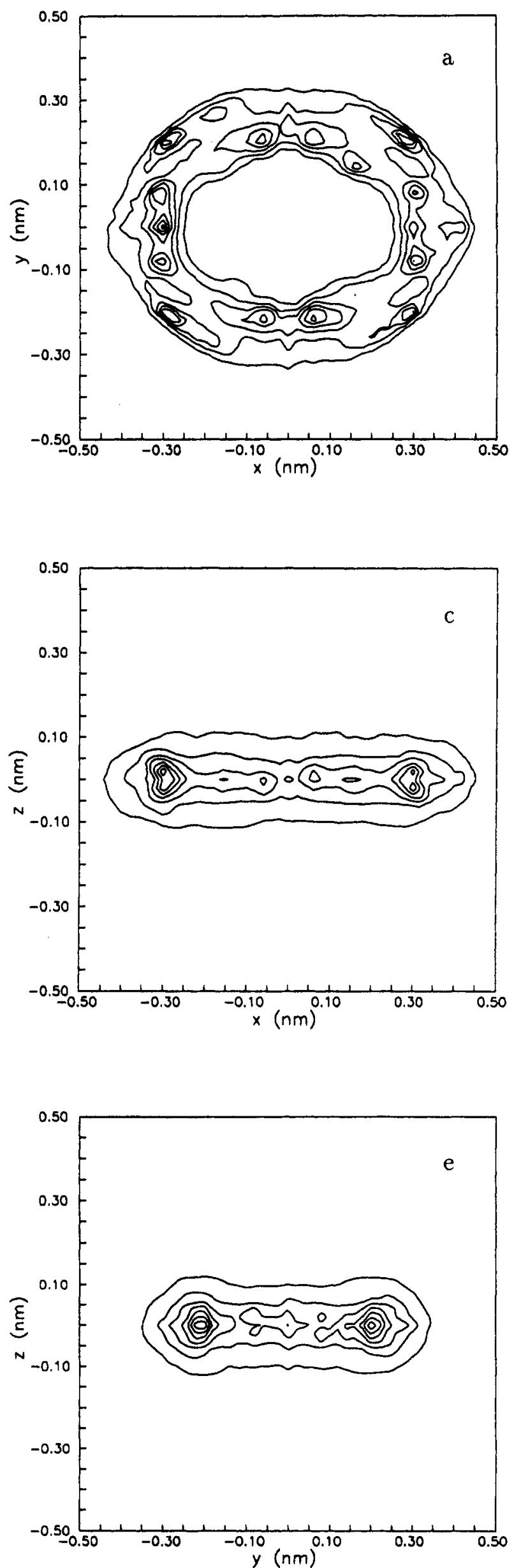

Leuwerink and Briels
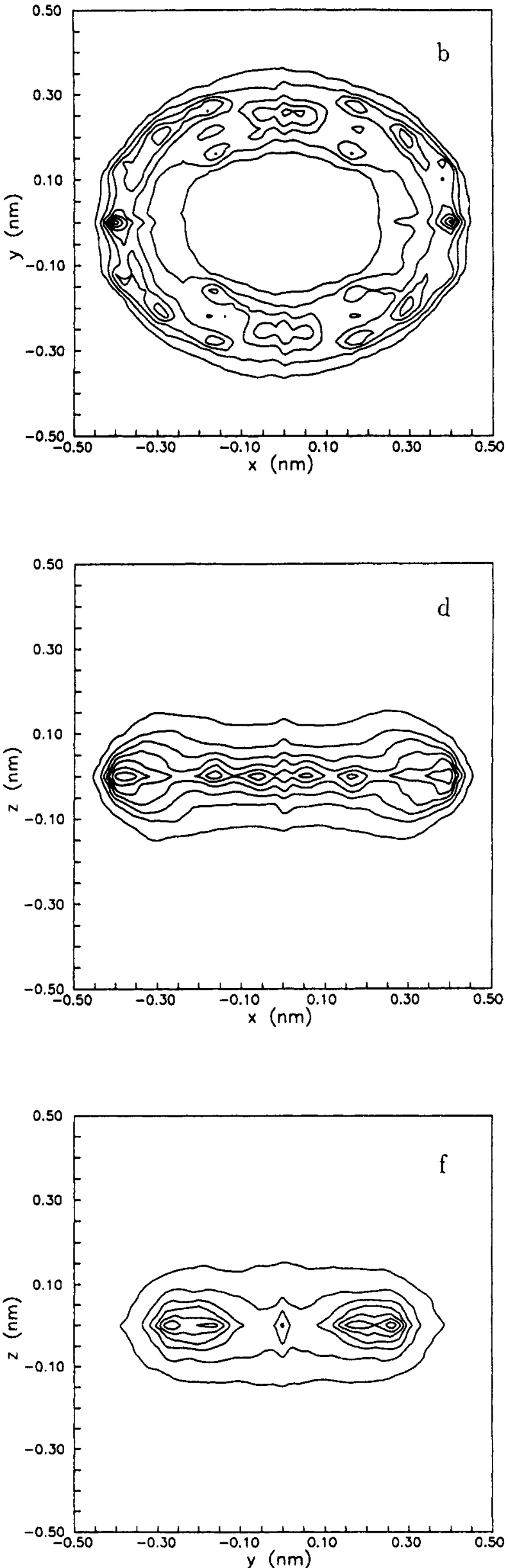

Figure 3. As Figure 2, but now for in vacuo simulation II (data from ref 23). 
18-Crown-6 in Cyclohexane
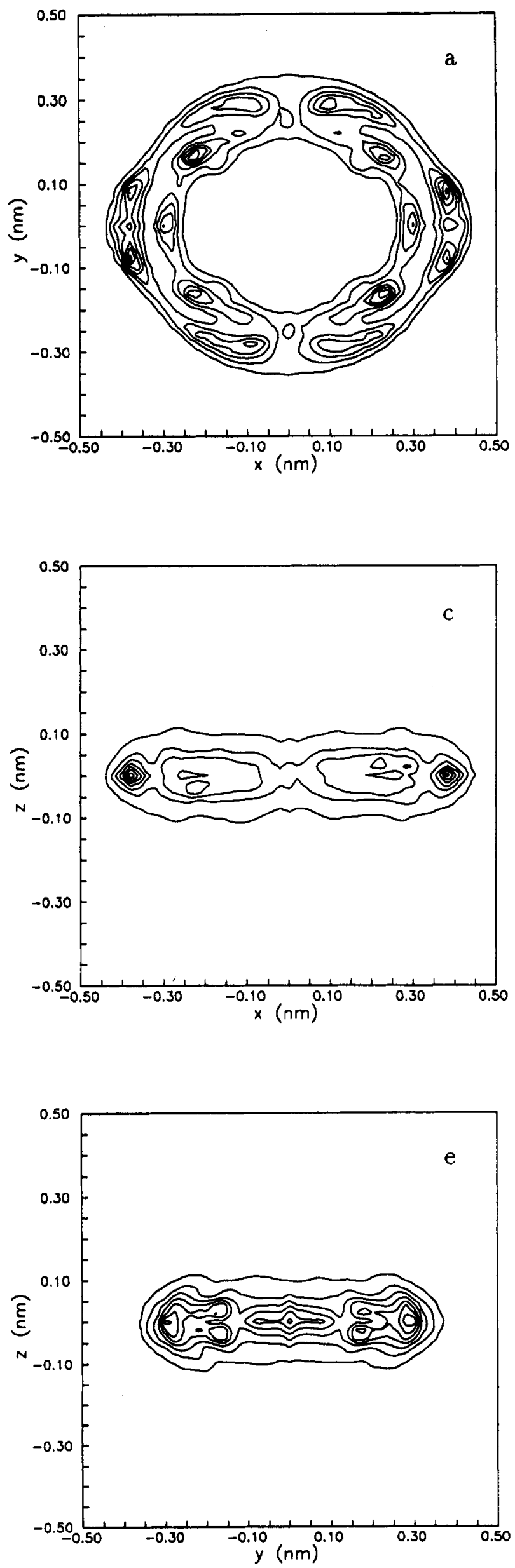

Figure 4. As Figure 2, but now for in vacuo simulation III.
J. Phys. Chem., Vol. 99, No. 45, 199516555
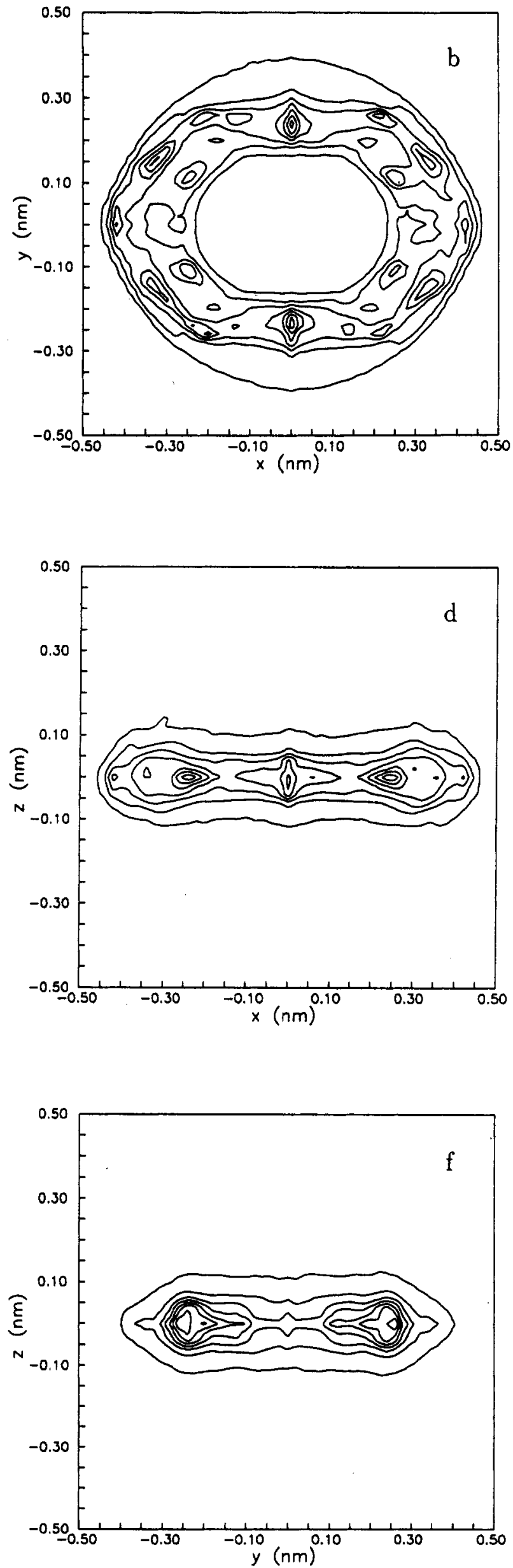

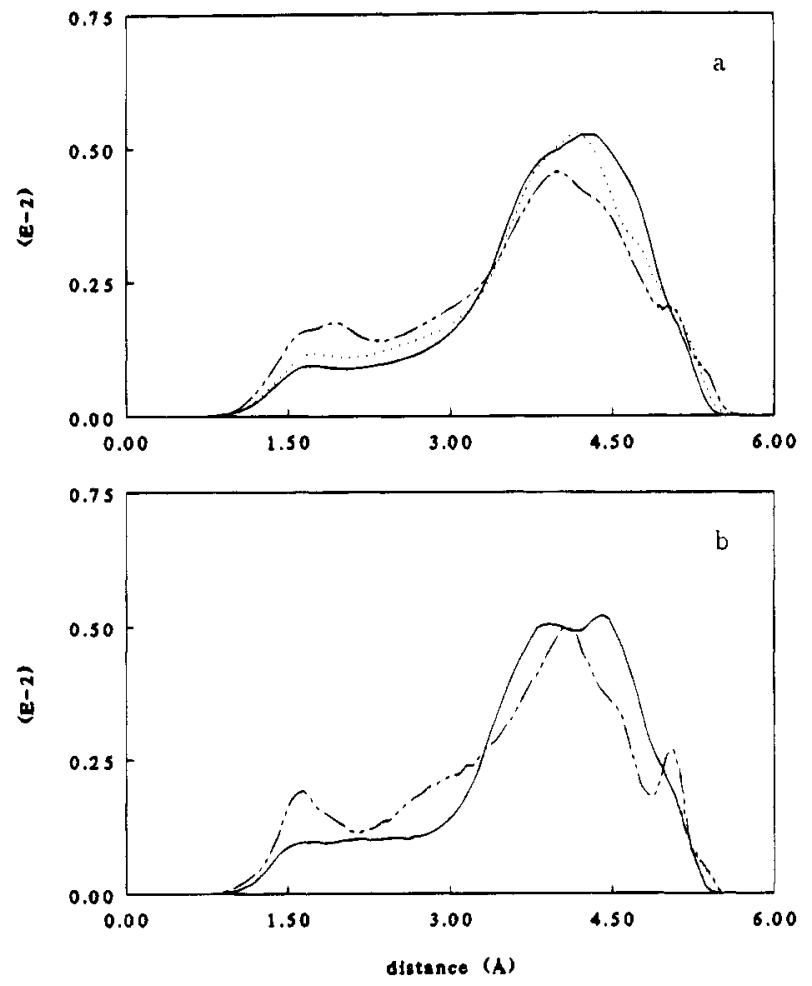

Figure 5. Distributions of distances of the hydrogen atoms of 18 crown- 6 to the center of mass of the molecule using potential model I (solid line), potential model II (dotted), and potential model III (dashdotted) for (a) for the in vacuo simulations and (b) for the cyclohexane solutions.

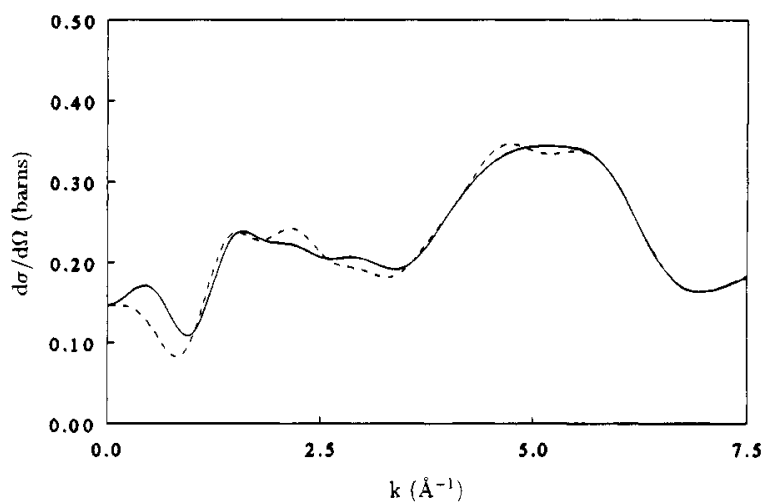

Figure 6. Neutron scattering cross sections calculated for 18-crown-6 in cyclohexane. Solid line corresponds with the simulation with potential model I (data from ref 23). The dashed line corresponds with the simulation using potential model III for the crown ether.

on atom $i$, and $g_{i j}(r)$ is the intramolecular radial distribution function for atoms $i$ and $j$ normalized to 1 . With eq 1 the dipole moments from section 4.1 were exactly reproduced.

Next, neutron scattering cross sections and X-ray intensities are determined. We only regard the 18 -crown- 6 molecule from the two cyclohexane solutions and neglect the influence of the solute on the solvent structure. For the neutron cross section can be written

$$
\begin{aligned}
\frac{\mathrm{d} \sigma}{\mathrm{d} \Omega}(k)=\frac{1}{N} \sum_{i} S_{i i} b_{i}^{2}+\frac{1}{N} \sum \sum_{i \neq j} S_{i j} b_{i} b_{j} \times & \\
& \left\{4 \pi \int_{0}^{\infty} r^{2} \mathrm{~d} r \frac{\sin (k r)}{k r} g_{i j}(r)\right\}
\end{aligned}
$$

where $b_{i}$ is the neutron scattering length of atom $i$. In Figure 6 the cross section is shown as a function of $k$ for both potential

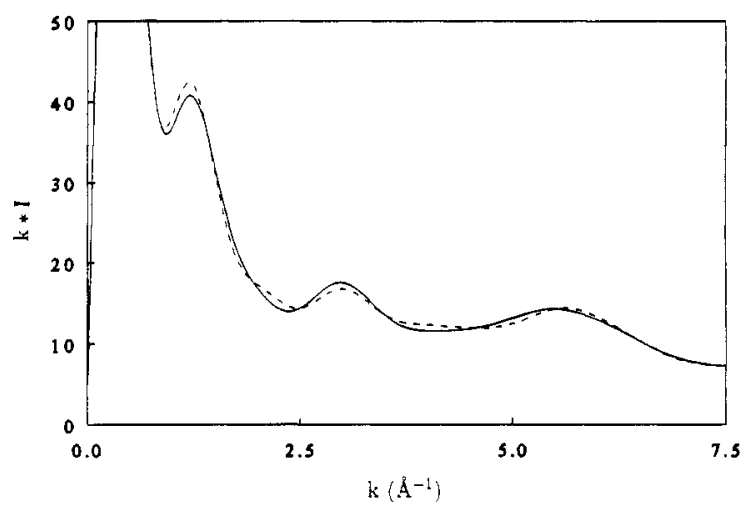

Figure 7. X-ray intensities (multiplied with $k$ ) calculated for 18crown-6 in cyclohexane. Solid and dashed lines correspond with the same simulations as in Figure 6.

models. For small angles $\theta(k=4 \pi / \lambda \sin \theta)$ the spectra differ. Neutron scattering experiments might acknowledge if indeed potential model III actually performs better as indicated by the calculated dipole moment. Similar experiments ${ }^{41}$ have been done on concentrated aqueous urea solutions, but the results were not very promising. ${ }^{42}$

Substitution of an atom type in the 18-crown- 6 molecule by one of its isotopes is not useful. The neutron scattering lengths of the isotopes of oxygen and carbon hardly differ. The differences between "normal" hydrogen and its isotopes deuterium and tritium on the other hand are so large that, combined with the fact that almost two-thirds of the molecule consists of this atom type, the effect of isotope substitution in this case will dominate the spectrum completely and conceal the differences between both potential models.

If in eq 2 the $b_{i}$ 's are replaced by the atomic scattering factors $f_{i}(k)$, we get the intensity as a function of $k$ as it can be measured from an X-ray experiment. The results from the calculation are shown in Figure 7. At $k=2.2$ and $4.0 \AA^{-1}$ the functions for model III show two weak shoulders, and it is doubtful whether the experimental resolution of an X-ray experiment is large enough to make a distinction between the potentials.

\section{Conclusions}

In this article we have investigated some properties of 18crown- 6 in vacuo and in cyclohexane, using a new charge model for the crown ether. These charges have recently been developed by Szentpály and Shamovsky. ${ }^{32}$

The root-mean-square dipole moments calculated from a gas phase simulation (3.02 D) and a cyclohexane solution (2.94 D) are considerably larger than previously reported theoretical dipole moments. These values are close to the value $(3.17 \mathrm{D})$ we have recently inferred from the experimental data in ref 23 . In spite of these good results some doubts about the present potential remain, especially because it does not have the crystallographic $C_{i}$ conformation as its global minimum and because the dipole moment of dimethyl ether calculated on the basis of the present charges seems to be rather large. We do believe however that the results of this paper prove the importance of atomic polarization effects and that the present model is about the best one can get with static charges.

The new charge model results in a molecular configuration distribution which differs from the ones obtained with all previous models. This is best appreciated by looking at the average mass distribution in the molecule, as we proposed in ref 23 . The molecule turns out to be a little more expanded compared to the average structure with other charge models. We suggest that a neutron scattering experiment may discrimi- 
nate between the diferrent potential models. Indeed, the neutron scattering function (Figure 6) is qualitatively different at small $k$ values for different potential models.

Supporting Information Available: Tables V, VI, and VII, listing the conformations of 18-crown- 6 with an occurrence of $0.5 \%$ or more from respectively in vacuo simulations I, II, and III ( 3 pages). Ordering information is given on any current masthead page.

\section{References and Notes}

(1) Pedersen, C. J. J. Am. Chem. Soc. 1967, 89, 2495

(2) Pedersen, C. J. Angew. Chem., Int. Ed. Engl. 1988, 27, 1021 and references therein. 146.

(3) Allen, F. H.; Kennard, O.; Taylor, R. Acc. Chem. Res. 1983, 16,

(4) Izatt, R. M.; Bradshaw, J. S.; Nielsen, S. A.; Lamb, J. D.; Christensen, J. J.; Sen, D. Chem. Rev. 1985, 85, 271. 1721 .

(5) Izatt, R. M.; Pawlak, K.; Bradshaw, J. S. Chem. Rev. 1991, 91,

(6) Billeter, M.; Howard, A. E.; Kuntz, I. D.; Kollman, P. A. J. Am. Chem. Soc. 1988, 110,8385 .

(7) Grootenhuis, P. D. J.; Kollman, P. A. J. Am. Chem. Soc. 1989 , 111,4046

(8) Dang, L. X.; Kollman, P. A. J. Am. Chem. Soc. 1990, 112, 5716. 5076 .

(9) van Eerden, J.; Harkema, S.; Feil, D. J. Phys. Chem. 1988, 92,

(10) Sun, Y.; Kollman, P. A. J. Chem. Phys. 1992, 97, 5108

(11) Ha. Y. L.; Chakraborty, A. K. J. Phys. Chem. 1991. 95, 10781.

(12) Kowall, T.; Geiger, A. J. Phys. Chem. 1994, 98, 6216.

(13) Hori, K.; Yamada, H.; Yamabe, T. Tetrahedron 1983, 39, 67.

(14) Bruning, H.; Feil, D. J. Comput. Chem. 1991, 12, 1.

(15) Rencsok, R.; Kaplan, T. A.; Harrison, J. F. J. Chem. Phys. 1993, 98,9758 .

(16) Thompson, M. A.; Glendening, E. D.; Feller, D. J. Phys. Chem. 1994, 98,10465 .

(17) Glendening, E. D.; Feller, D.; Thompson, M. A. J. Am. Chem. Soc.

1994. $116,10657$.

(18) Wipff, G.; Weiner, P.; Kollman, P. A. J. Am. Chem. Soc. 1982, 104,3249 .
(19) Uiterwijk, J. W. H. M.; Harkema, S.; van de Waal, B. W.; Göbel F.; Nibbeling, H. T. M. J. Chem. Soc., Perkin Trans. 2 1983, 1843.

(20) Straatsma, T. P.; McCammon, J. A. J. Chem. Phys. 1989, 91, 3631.

(21) Sun, Y.; Kollman, P. A. J. Comput. Chem. 1992, 13, 33.

(22) Troxler, L.; Wipff, G. J. Am. Chem. Soc. 1994, 116, 1468.

(23) Leuwerink, F. T. H.; Briels, W. J. J. Chem. Phys., in press.

(24) Perrin, R.; Decoret, C.; Bertholon, G.; Lamartine. R. Nouv. J. Chim. $1983,7,263$.

73 .

25) Caswell, L. R.; Suvannunt, D. S. J. Hetrocycl. Chem. 1988, 25,

(26) Hase, W. L.; Richou, M. C.: Mondro, S. L. J. Phys, Chem. 1989. $93,539$.

(27) van Eerden, J.; Briels, W. J.; Harkema, S.; Feil, D. Chem. Phys. Lett. 1989, 164, 370.

(28) Mazor, M. H.; McCammon, J. A.; Lybrand, T. P. J. Am. Chem Soc. $1989,111,55$

(29) Mazor, M. H.; McCammon, J. A.; Lybrand, T. P. J. Am. Chem Soc. $1990,112,4411$.

(30) Welti, M. Ph.D. Thesis, ETH Zürich, 1987.

(31) Singh, U. C.; Kollman, P. A. J. Comput. Chem. 1984, 5, 129.

(32) von Szentpály, L.; Shamovsky, I. L. J. Mol. Struct. (THEOCHEM) 1994, $305,249$.

(33) van Gunsteren, W. F.; Berendsen, H. J. C. Groningen Molecular Simulation Library, Groningen, The Netherlands, 1987.

(34) Weiner, S. J.; Kolmann, P. A.; Nguyen, D. T.; Case, D. A. J. Comput. Chem. 1986, 7, 230.

(35) Harris, J. G.; Stillinger, F. H. J. Chem. Phys. 1991, 95, 5953.

(36) McClellan, A. L. Tables of Experimental Dipole Moments; W. H. Freeman: San Francisco, 1963.

(37) Böttcher, C. J. F.; van Belle, O. C.; Bordewijk, P.; Rip, A. Theory of Electric Polarization: Elsevier: Amsterdam. 1973: Vol. 1.

(38) von Szentpály, L.; Shamovsky, I. L.; Nefedova, V. V.; Zubkus, V. E. J. Mol. Struct. (THEOCHEM) 1994, 308, 125.

(39) Letcher, T. M.; Paul, J. J.; Kay, R. L. J. Solution Chem. 1991, 20 1001 .

(40) Letcher, T. M.; Mercer-Chalmers, J. D.; Kay, R. L. Pure Appl. Chem. 1994, 66, 419.

(41) Finney, J. L.; Turner, J. Electrochim. Acta 1988, 9, 1183.

(42) Boek, E. S.; Briels, W. J. J. Chem. Phys. 1993, 98, 1422.

JP951223S 